\title{
Memristor based ring oscillators true random number generator with different window functions for applications in cryptography
}

\author{
Noor Alia Nor Hashim, Julius Teo Han Loong, Azrul Ghazali, Fazrena Azlee Hamid \\ College of Engineering, Universiti Tenaga Nasional, Malaysia
}

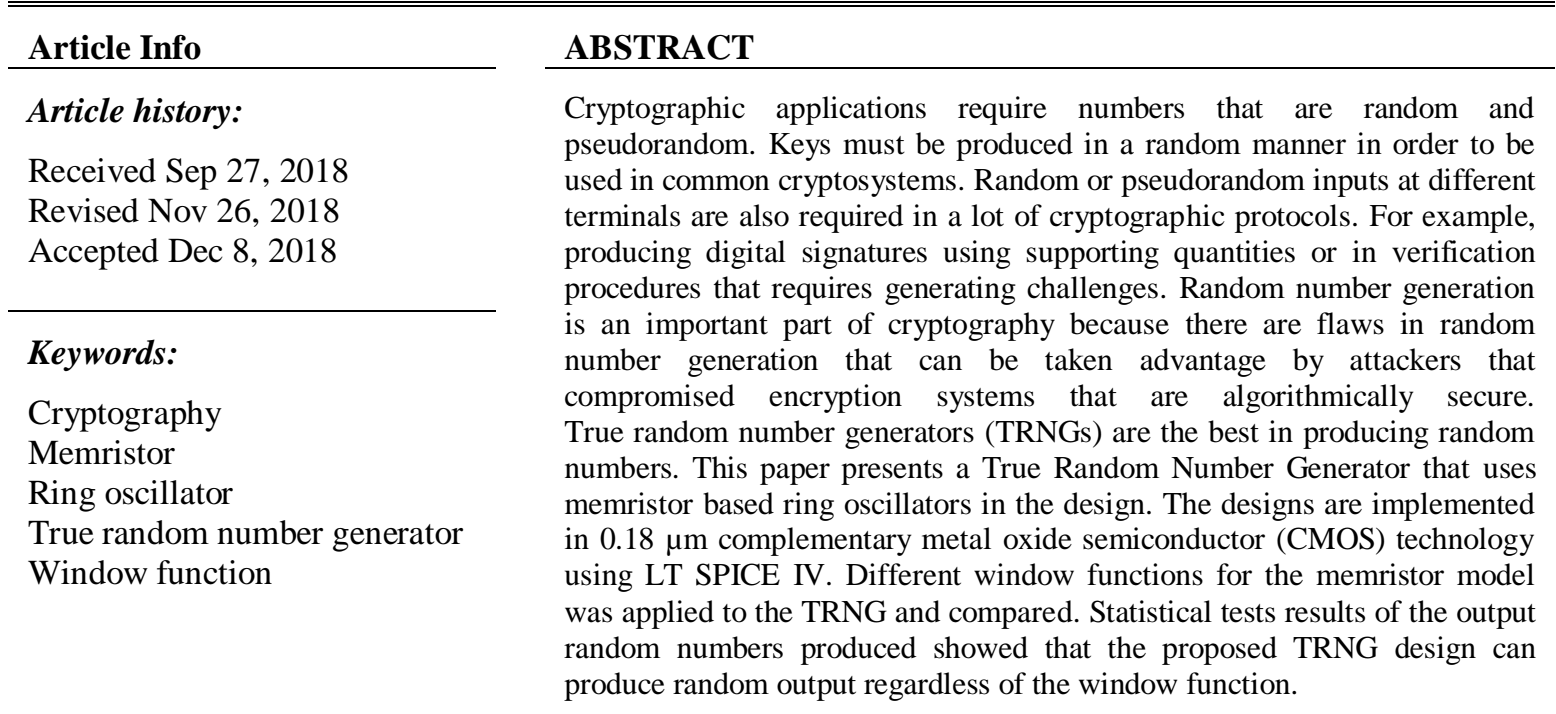

Copyright () 2019 Institute of Advanced Engineering and Science. All rights reserved.

\section{Corresponding Author:}

Noor Alia Nor Hashim,

College of Engineering,

Universiti Tenaga Nasional,

Putrajaya Campus, Jalan IKRAM-UNITEN, 43000, Kajang, Selangor, Malaysia.

Email: noor.alia@uniten.edu.my

\section{INTRODUCTION}

Nowadays, there are a lot of multi-level attacks that can affect the hardware and software implementations of the cryptographic algorithms. It is very important in every section of the modern cryptography to have a series of random numbers. Some solutions to ensure hardware security are counterfeiting prevention using physically unclonable functions (PUFs) and true random number generator. The difference between a PUF and TRNG is that PUFs generates identical responses that look random for each challenge whereas a TRNG produces unique output bits. The randomness of the output can be increased by using memristor in the true random number generator (TRNG) design. Memristors have characteristics that are suitable to be implemented or leveraged for security. Memristors and resistive random-access memories (RRAMs) store information as resistance rather than charge, as other memories do. Memristor can be used as a replacement of some or all resistors in ring oscillators as the response is quite similar. TRNGs can be built using oscillators which has a simple design and an effective method. A ring oscillator (RO) is a type of relaxation oscillator that contains an odd number of inverters creating a non-sinusoidal signal alternating between a high and low voltage. Ring oscillator is simply made out of a number of inverters and the first inverter is connected to the output of the last inverter. This paper will focus on a memristor based ring oscillator TRNG design that can be applied in hardware security. 
For the purpose of gaining design insights, this paper focuses on the TRNG design using CMOS technology and how the memristor can be implemented in the design as well as the effects it has on the random characteristics of the output bits generated when the memristor model adopts different window functions. The memristor-based TRNG designs are modeled using three different window functions; Prodromakis, Joglekar and Biolek. Section 2 explains the review of TRNGs. Section 3 will explain the structural background of circuits and the modifications made to the TRNG design. Section 4 discusses the methodology of the research. Section 5 discusses the performance parameters of the TRNG design and the statistical test results for each window function using the National Institute of Standards and Technology (NIST) test suite. Finally, Section 6 concludes the paper.

\subsection{True Random Number Generator}

There are lots of applications such as encryption and random key generation that needs to use true random number generators (TRNGs) [1]. These generators use randomness that appears in physical phenomena, for example, thermal noise generated by resistors, noise generated by semiconductors, jitter in ring oscillators or randomness that results from nuclear decay [2]. It is very important to upgrade the security and result of these applications through an effective random number generation. This is because encrypted Internet networks can be hacked and break through by various attacks from users [3]. The randomness source used was relatively deterministic than truly unique and random in early designs and this made it vulnerable to multiple attacks. It is vital to produce a true randomness due to this problem.

\subsection{Memristor Modeling}

According to a circuit theorist, Leon Chua, there is a connection between the electric charge and magnetic flux linkage described as an electrical element that is non-linear and two-terminal. He discovered memristor in 1971 that proposed the missing relationship and envisioned the concept [4]. Memristor's resistance relies on the amount of current that earlier flowed through the circuit and is not constant according to the principal mathematical relations. In other words, the present resistance is determined on the electric charge and the direction that previously flowed as the circuit will retain its past amount. Even though there is no stream of electric power, the existing amount of the resistance of the memristor will be retained. Chua [4] stated that the memristor is the missing element for all types of twoterminal non-volatile electrical component that are centered on resistance changing effects but there are a lot of opposing arguments to this statement.

Memristors are compatible to be used in the fabrication of the CMOS processes. It has memory-like properties and are able to change its memresistance value based on its input [5]-[7]. The behaviour of a memristor is that it has an I-V plot of a hysteresis loop that lessen at the source as it is stimulated by a periodic signal [8]. This means that it will behave linearly as the frequency increases and nonlinearly when the frequencies are small as shown in Figure 1.

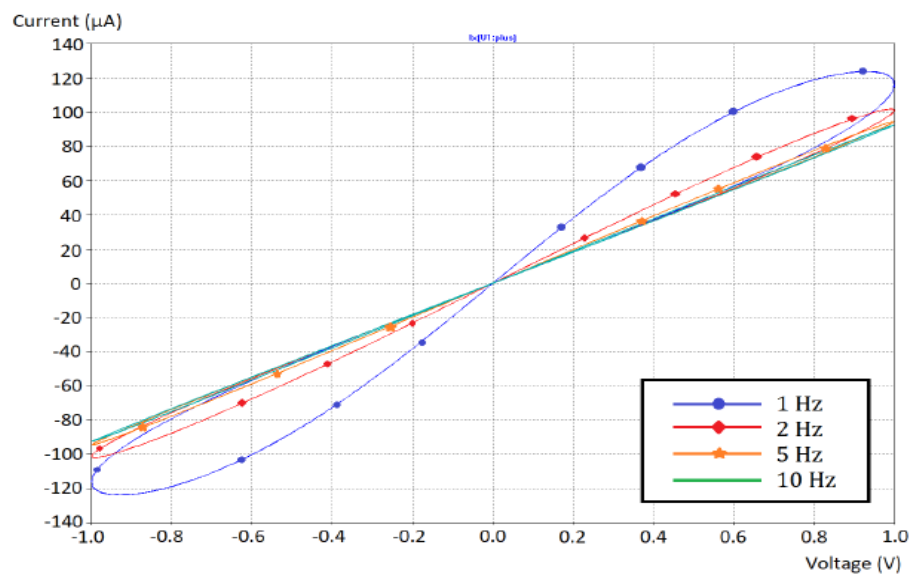

Figure 1. The I-V plot the memristor. 
In this paper, a memristor based ring oscillator TRNG will be designed and analyzed for security hardware application. The memristor used in the proposed TRNG design is a nonlinear ion drift model. The values of the memristor parameters are displayed below [9];

a) Resistance in ON state, $\mathrm{R}_{\mathrm{ON}}=100 \Omega$

b) Resistance in OFF state, $\mathrm{R}_{\mathrm{OFF}}=16 \mathrm{k} \Omega$

c) Resistance at $\mathrm{T}=0, \mathrm{R}_{\mathrm{INIT}}=11 \mathrm{k} \Omega$

d) Width of the thin film, $\mathrm{D}=10 \mathrm{~nm}$

e) Ion drift migration coefficient, $\mu=10 \mathrm{fm}^{2} /(\mathrm{V} \cdot \mathrm{s})$

f) Parameter of the WINDOW-function for modeling nonlinear boundary conditions, $\mathrm{p}=10$

\subsection{Window Functions for Memristor}

There are also different window functions that can be chosen for the memristor. The use of window functions is to produce a range of state variable and the nonlinearity near boundaries to make it stretch to zero when the state variable is at the limit by multiplying the window functions with the derivative of the state variable [10]. There are three different types of window functions that this paper will focus on; Joglekar, Biolek, and Prodromakis. All of the window functions fit the linear and nonlinear ion drift and TEAM memristor model except for TEAM window function which was designed to fit the Simmons tunneling barrier fitting memristor model. Below is a brief explanation for each window functions [10];

a) Joglekar

A generic symmetric window function introduced by Joglekar and Wolf that regulates the nonlinearity of the function by having a control value [11]

$$
f(x)=1-(2 x-1)^{2 p}
$$

b) Biolek

This window function solves the Joglekar's window function modeling imprecision and presented a PSPICE model for the memristor [12].

$$
f(x)=1(x-\operatorname{sgn}(-i))^{2 p}
$$

where $p$ is a positive integer, $i$ is the memristor current and $\operatorname{sgn}(i)=1$ when $i \leq 0$ and $\operatorname{sgn}(i)=0$ when $i<0$. c) Prodromakis

In Prodromakis et al. [13], this window function was designed to solved scalability and includes HP's window function.

$$
f(x)=j\left(1-\left[(x-0.5)^{2}+0.75\right]^{p}\right)
$$

where $p$ is a control value and a positive real number and $j$ is a scalable factor used to determine the maximum amount of window function $f_{\max }$.

\section{REVIEW OF TRNG}

TRNGs can be build using ring oscillators that has a simple and effective design. Ring oscillators are built using inverters that are odd in numbers and produce a non-sinusoidal signal that oscillates from a low voltage to a high voltage alternatingly [14]. The inverters are connected in a ring configuration by connecting the output of the inverter to the input of the next inverter continuously to build the ring oscillator. Ring oscillators possessed great characteristics such as it has a simple design, needs less power and low operating voltage [15]. The circuit will generate thermal noise and this will impact the vibrations of the ring oscillators output and make the output to be produced in a random manner. The vibrations is called jitter when in the time domain whereas it is called the phase noise when in the frequency domain. The source of entropy that we are focusing on is the jitter that occurs in the memristor based ring oscillator TRNG [16]. The proposed TRNG design in this paper is based on the work of Ning et al. [16] which uses digital design method and without difficult calibration. As shown in Figure 2, the TRNG adopts a topology that consists of ring oscillators, binary XOR-tree which is represented by the plus sign symbol, harvesting mechanism and post processing. 


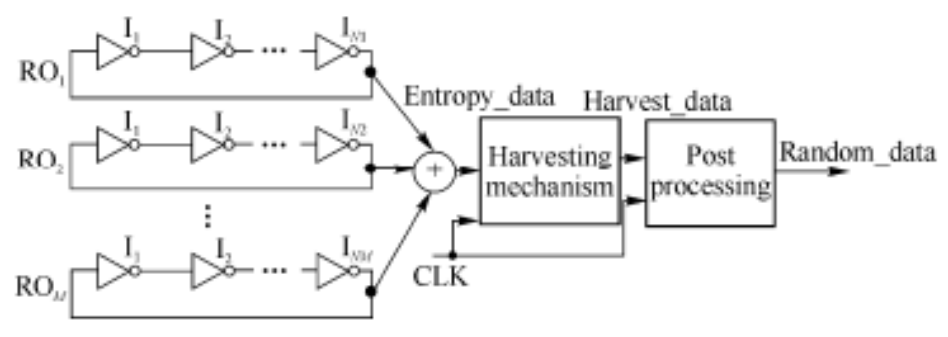

Figure 2. Topology of true random number generator [16]

\section{TOPOLOGY OF MEMRISTOR BASED RING OSCILLATORS TRNG}

This section explains the topology of the proposed memristor based ring oscillators TRNG design which comprises of entropy source, harvesting mechanism and post processing. This design is based on the design proposed by Ning et al. [16] that adopts a varied range of supply voltage, high speed TRNG that uses ring oscillators made by prime-length numbers of ring oscillators. Their design introduces a simple Von Neumann corrector that can improve the output randomness. The proposed TRNG differs from Ning et al. TRNG by adopting the concept of a common source stage with a resistive load to build the inverter and having memristor replacing the resistor part of the inverter. The ring oscillators in the entropy source circuit are based on the inverters that implements memristor.

\subsection{Entropy Source}

The main part of the TRNG is the entropy source circuit. The entropy exists in the randomness that occurs in the phase noise of the memristor based prime-length ring oscillators. This entropy is extracted and sampled from the output of the ring oscillators. There are 4 sets of memristor based ring oscillators (M-RO) and each one comprises of 13,17, 23 and 31 number of inverters respectively and is all in a ring configuration. The M-ROs are then connected to 3 XORs. Figure 3 shows the components that comprise the circuit. The overlaps in the transition zones are decreased while the entropy increased by adopting primelength inverters to build the ring oscillators. For each of the ring oscillator, memristor was implemented in each stage in the entropy source circuit. The inverters adopt the concept of a common source stage with a resistive load as its single stage. The resistor is then replaced by a memristor as can be seen in Figure 4. The entire ring oscillators in the entropy source circuit were designed using the memristor based common source as implemented in the inverter and was compared and analyzed to find the best window function to adopt in the design.

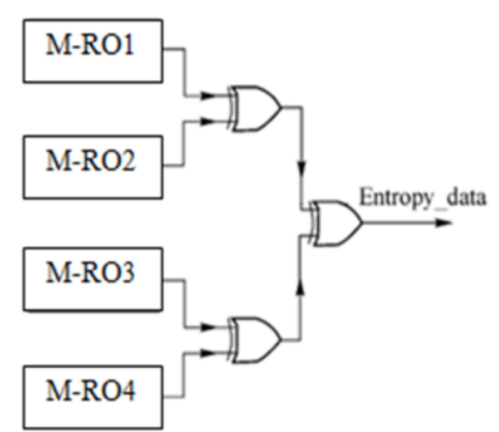

Figure 3. Effects of selecting different switching under dynamic condition

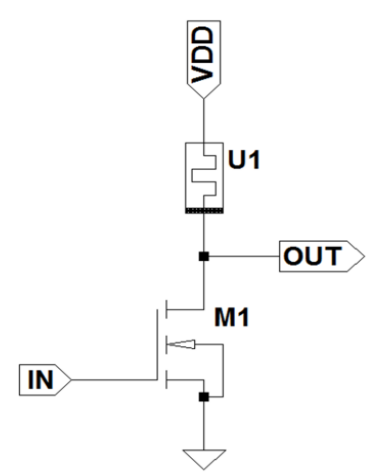

Figure 4. Memristor based common source circuit

\subsection{Harvesting Mechanism}

The harvesting mechanism is constructed using a set/reset (SR) latch that makes up a simple arbiter and is shown in Figure 5. A latch or a flip-flop is a circuit that possesses two steady states and has a purpose of storing state information. A flip-flop is a bistable multivibrator. By supplying signals to the control inputs, the state of the circuit can be varied and can have one or two outputs. 


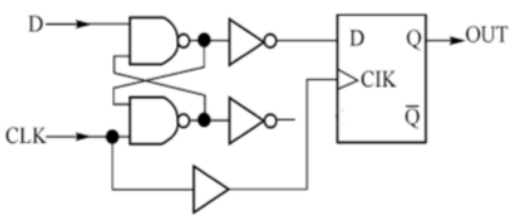

Figure 5. SR-latch based arbiter harvesting mechanism circuit

\subsection{Post Processing}

The randomness of the data output can be improved by adopting post processing that is build using a simple Von Neumann corrector. Figure 6 shows the structure of the Von Neumann corrector which consists of a number of logic gates. The output bit stream will be modified by eliminating bit streams of 00 and 11 and turning 01 to 1 and 10 to 0 . This will result in the output to be reduced by a factor of 4 , producing a 1 bit from a 4 bit.

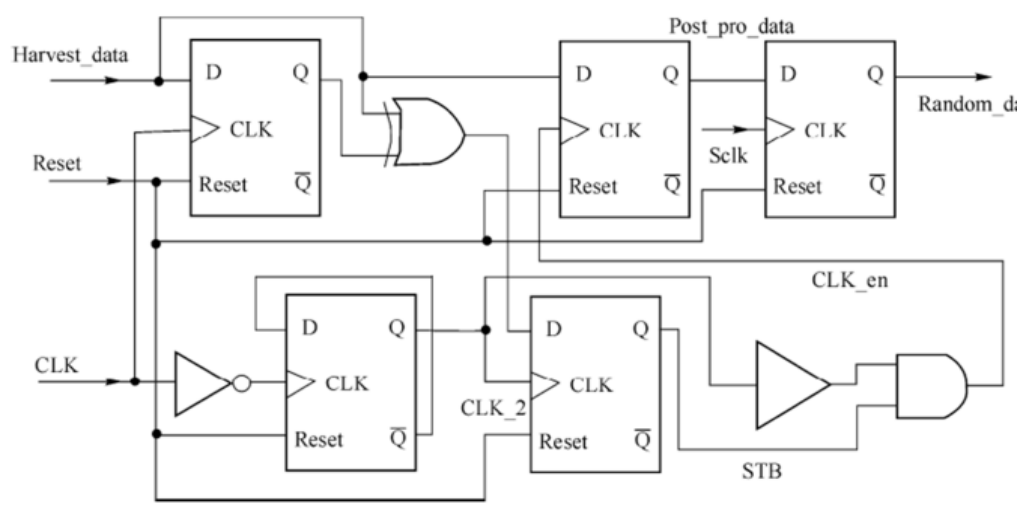

Figure 6. Simple von neumann corrector [16]

\section{RESEARCH METHOD}

This section explained the methodology of the research that comprises of the simulation setup for the design, performance criteria that is being used to evaluate the randomness characteristics of the output bits and the comparison of different window functions used. The memristor based ring oscillators TRNG design is based on Ning et al. [16] work as shown in Figure 1. A sample of the final random output that has been harvested from the entropy of jitter source is shown in Figure 7. The entropy data is produced as a result of the four memristor based ring oscillators (M-RO) outputs and goes through the harvesting mechanism circuit to produce the harvest data. This output data will be sampled to the post processing circuit to produce the final result of the random data.

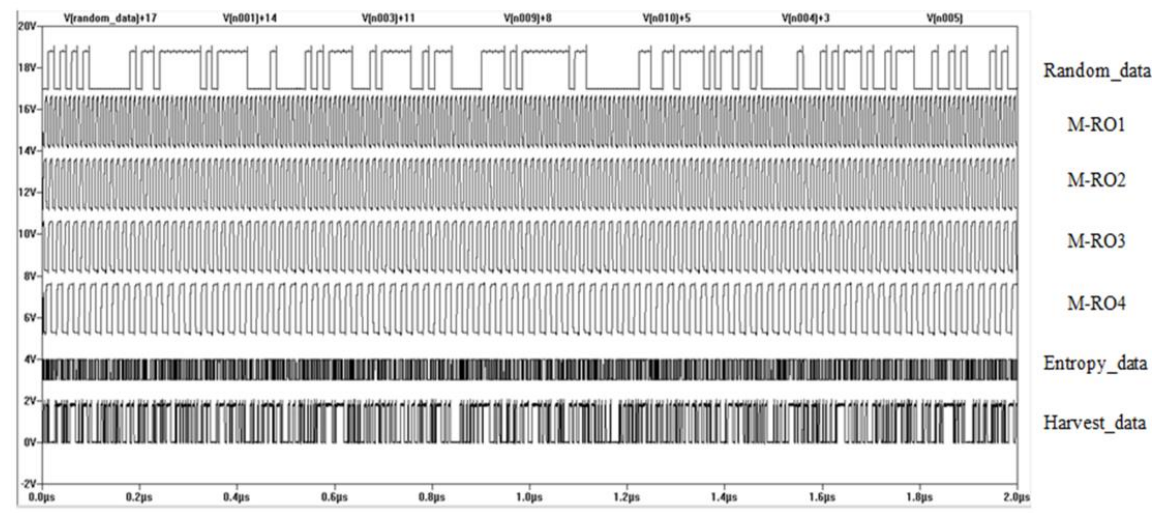

Figure 7. Memristor based ring oscillators TRNG output

Memristor based ring oscillators true random number generator with different... (Noor Alia Nor Hashim) 


\subsection{Simulation Setup}

The proposed TRNG design was simulated using LTspice IV which is by Linear Technology Corporation, on SilTerra 180nm CMOS process. The random output bits produced had a length of 167 bits. The circuit simulation software was simulated on Microsoft Windows 7 with Intel i5 core operating at 2.67GHz and 4GB RAM.

\subsection{National Institute of Standards and Technology (NIST)}

The obtained bits represented by random data were tested using National Institute of Standards and Technology (NIST) test suite [13] to see whether the output bits are considered random. Comparison of the test results were made for sample bits of 167 bits in length. The NIST test suite contains 15 statistical tests that are used to verify the randomness characteristics of binary bit streams that are generated by true or pseudorandom number generators. A value of probability (P-value) for the null hypothesis is produced based on the binary sequence tested. To pass a specific test, the P-value must be more than 0.01 [17]. This paper will only focus on nine out of the 15 tests available. Below are the nine tests that this paper will focus on:
a) The Frequency (Monobit) Test
b) Frequency Test within a Block
c) The Runs Test,
d) Tests for the Longest-Run-of-Ones in a Block
e) The Discrete Fourier Transform (Spectral) Test
f) The Overlapping Template Matching Test
g) The Serial Test
h) The Approximate Entropy Test
i) The Cumulative Sums (Cusum) Test

\section{RESULTS AND ANALYSIS}

\subsection{Circuit Parameters}

Table 1 shows the circuit parameters for all of the TRNG design using different window functions of Prodromakis, Joglekar and Biolek.

Table 1. Circuit Parameters

\begin{tabular}{lccc}
\hline Performance Parameters & Prodromakis & Joglekar & Biolek \\
\hline Fabrication Technology & $0.18 \mu \mathrm{m}$ CMOS process & $0.18 \mu \mathrm{m}$ CMOS process & $0.18 \mu \mathrm{m}$ CMOS process \\
Supply voltage & $2.6 \mathrm{v}$ & $1.8 \mathrm{v}$ & $1.8 \mathrm{v}$ \\
Power Consumption & $626.63 \mu \mathrm{w}$ & $433.82 \mu \mathrm{w}$ & $433.82 \mu \mathrm{W}$ \\
Output Throughput & $83.4 \mathrm{Mbit.} \mathrm{s}^{-1}$ & $83.4 \mathrm{Mbit.} \mathrm{s}$ & $83.4 \mathrm{Mbit}^{-1} \mathrm{~s}^{-1}$ \\
\hline
\end{tabular}

\subsection{Different Window Functions for Memristor Model}

The memristor based ring oscillators TRNG were simulated with different window functions for the memristor model to produce random output bits. The window functions that were implemented were from Prodromakis, Joglekar and Biolek. All of the designs were simulated using LTspice IV which is by Linear Technology Corporation, on SilTerra 180nm CMOS process. This section shows the performance results of the NIST tests for all of the TRNG designs. Each memristor based ring oscillators TRNG have a 167 response bits in length. Table 2 displays the statistical test results for the memristor based TRNG after the output bits were subjected to the NIST test suite. The output passed all of the tests chosen of the NIST test suite.

Table 2. Nist Test for $0.18 \mu \mathrm{m}$ CMOS Technology Memristor based TRNG

\begin{tabular}{llccccc}
\hline \multicolumn{1}{c}{ Statistical Test } & $\begin{array}{c}\text { P-value } \\
\text { (Prodromakis) }\end{array}$ & $\begin{array}{c}\text { Pass/ } \\
\text { Fail }\end{array}$ & $\begin{array}{c}\text { P-value } \\
\text { (Joglekar) }\end{array}$ & $\begin{array}{c}\text { Pass/ } \\
\text { Fail }\end{array}$ & $\begin{array}{c}\text { P-value } \\
\text { (Biolek) }\end{array}$ & $\begin{array}{c}\text { Pass/ } \\
\text { Fail }\end{array}$ \\
\hline Frequency & 0.588042 & Pass & 0.036679 & Pass & 0.486153 & Pass \\
Block Frequency & 0.859684 & Pass & 0.013328 & Pass & 0.033895 & Pass \\
Cusum-Forward & 0.376526 & Pass & 0.032893 & Pass & 0.073357 & Pass \\
Cusum-Reverse & 0.837766 & Pass & 0.073357 & Pass & 0.327252 & Pass \\
Runs & 0.956319 & Pass & 0.034313 & Pass & 0.011622 & Pass \\
Long Runs of Ones & 0.449257 & Pass & 0.451605 & Pass & 0.209211 & Pass \\
Spectral DFT & 0.098738 & Pass & 0.098738 & Pass & 0.346759 & Pass \\
NonOverlapping Templates & 1.000000 & Pass & 1.000000 & Pass & 1.000000 & Pass \\
Approximate Entropy & 1.00000 & Pass & 1.000000 & Pass & 1.000000 & Pass \\
Serial & 0.937791 & Pass & 0.498961 & Pass & 0.498961 & Pass \\
\hline
\end{tabular}


Table 3 shows the different TRNGs existed and the proposed TRNG design in comparison of its circuit parameters. The TRNG design using Prodromakis window function has the highest power consumption with $626.63 \mu \mathrm{w}$ compared to Joglekar and Biolek with $433.82 \mu \mathrm{w}$. This is due to the minimum supply voltage of $2.6 \mathrm{v}$ that the design needs in order to make sure all of the transistors of the inverters are in saturation. The TRNG design passed all of the chosen NIST tests regardless of the window function being used and showed that it has randomness characteristics in the output bits produced. Hence, the TRNG design can implement any of the window functions to produce a random output.

Table 3. Existing and Proposed TRNGs

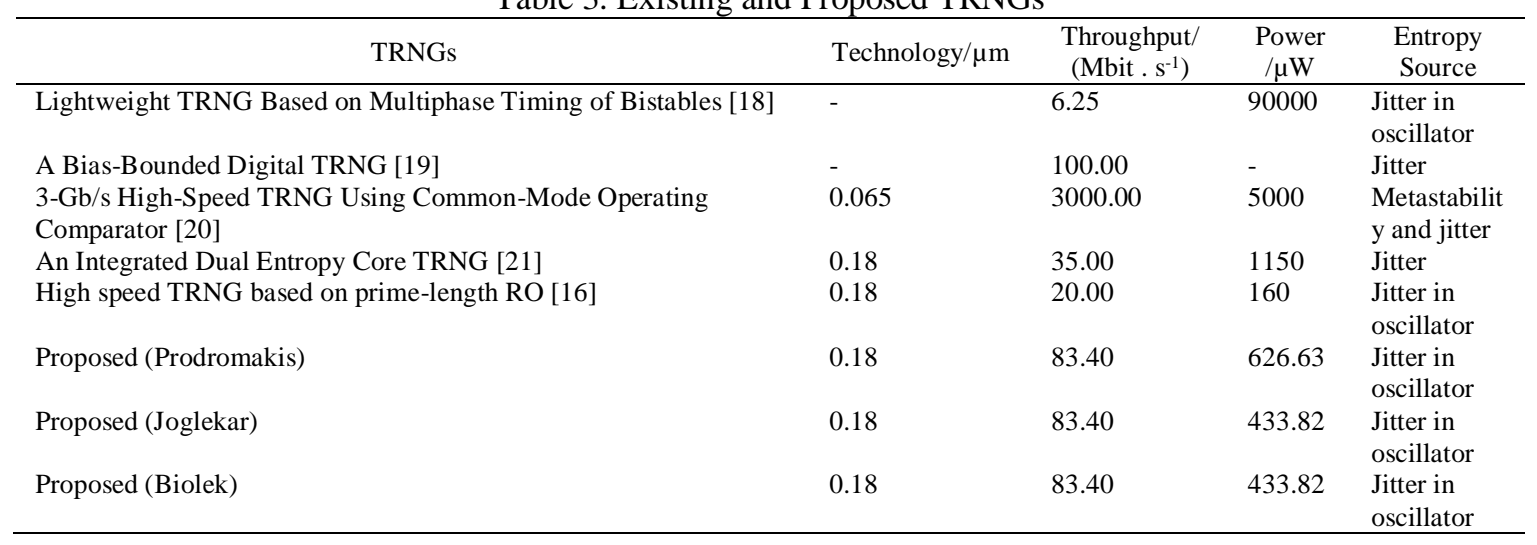

This is as predicted because although the TRNG adopts memristor using different window functions, the oscillation frequencies of the ring oscillators in the design are in the high frequency range of around $100 \mathrm{MHz}$ [15]. One of the main characteristic of a memristor is that it acts linearly at high frequencies. The I-V plot of a memristor is a hysteresis loop that diminishes to a straight line as the frequency increases [8]. In summary, the memristor acts like a fixed resistor when operating in high frequencies as the memresistance changes little in its value. Besides that, all of the window functions of the memristor has the same linear ion drift model. They act typically the same with exception of their boundary conditions.

\section{CONCLUSION}

This paper presents a memristor based ring oscillators TRNG design that has been implemented in the $0.18 \mu \mathrm{m}$ complementary metal oxide semiconductor (CMOS) technology using LT SPICE software. The proposed TRNG design consists of various numbers of prime lengths of inverters in the ring oscillators of the entropy source circuit, a harvesting mechanism that was designed using SR-latch based arbiter and adopted a post processing design based on a Von Neumann corrector. The modifications were made in the entropy source circuit where memristors was implemented in the inverters. All of the designs were subjected to the NIST test suite and passes all of the chosen tests. This proves that the design can produce output bits with randomness characteristics regardless of the window functions being used in the memristor model.

\section{ACKNOWLEDGEMENTS}

This research was sponsored and supported under the FRGS grant for project FRGS/1/2015/TK04/ UNITEN/02/2 awarded by Ministry of Higher Education, Malaysia and by the Universiti Tenaga Nasional (UNITEN) Internal Grant under the project code J510050761. Many thanks to my colleagues from Universiti Tenaga Nasional who provided their insight and expertise during the research.

\section{REFERENCES}

[1] M. Stipcevic and S. K. Koc, " True Random Number Generators", Institute Zagreb, Croatia \& University of California, Santa Barbara, USA, 2014.

[2] V. Kote, V. Molata, J. Jakovenko, "Improved Structure of True Random Number Generator with Direct Amplification of Analog Noise", Department of Microelectronics, Faculty of Electrical Engineering, Prague, 2012.

[3] S. Robson, B. Leung, and G. Gong, "Truly Random Number Generator Based on a Ring Oscillator Utilizing Last Passage Time," IEEE Transactions on Circuits and Systems II: Express Briefs, vol. 61, pp. 937-941, 2014.

[4] L. O. Chua, "Memristor - the missing circuit element," IEEE Trans.Circuit Theory, vol. 18, pp. 507-519, // 1971. 
[5] G. S. Rose, M. Uddin, and M. B. Majumder, "A designer's rationale for nanoelectronic hardware security primitives," in Proceedings of IEEE Computer Society Annual Symposium on VLSI, ISVLSI, 2016, pp. 194-199.

[6] G. S. Rose, N. McDonald, L. K. Yan, B. Wysocki, and K. Xu, "Foundations of memristor based PUF architectures," in 2013 IEEE/ACM International Symposium on Nanoscale Architectures (NANOARCH), 2013, pp. 52-57.

[7] K.-T. T. Cheng and D. B. Strukov, "3D CMOS-memristor hybrid circuits: devices, integration, architecture, and applications," presented at the Proceedings of the 2012 ACM international symposium on International Symposium on Physical Design, Napa, California, USA, 2012.

[8] L. O. Chua and S. M. Kang, "Memristive devices and systems," Proc. IEEE, vol. 64, pp. 209-223, 1976.

[9] Z. Biolek, V. Biolkova, and D. Biolek, "SPICE model of memristor with nonlinear dopant drift," Radioengineering, 2009.

[10] M. E. F. A.G. Radwan, "Chapter 2 Memristor: Models, Types and Applications," Studies in Systems, Decision and Control 26, Springer International Publishing Switzerland, 2015.

[11] Joglekar, Y., Wolf, S., "The elusive memristor: properties of basic electrical circuits",: Eur. J. Phys. 30(4), $661,2009$.

[12] Biolek, D., Biolek, Z., Biolkova, V., "SPICE modeling of memristive, memcapacitative and meminductive systems",: In: European Conference on Circuit Theory and Design ECCTD 2009, pp. 249-252. IEEE 2009.

[13] Prodromakis, T., Peh, B.P., Papavassiliou, C., Toumazou, C., “A versatile memristor model with nonlinear dopant kinetics", : IEEE Trans. Electron Devices 58(9), 3099, 2011.

[14] H. Masten, " Ring oscillator design in 32nm CMOS with frequency and power analysis for changing supply voltage", Department of Electrical Engineering and Computer Science, Auburn University.

[15] M. Mndal and B. Sarkar, "Ring oscillators: Characteristics and applications," Indian Journal of Pure and Applied Physics, vol. 48, pp. 136-145, February 2010.

[16] L. Ning, J. Ding, B. Chuang, and Z. Xuecheng, "Design and validation of high speed true random number generators based on prime-length ring oscillators," The Journal of China Universities of Posts and Telecommunications, vol. 22, pp. 1-6, 2015/08/01 2015.

[17] A. Rukhin et. al., "A Statistical Test Suite for Random and Pseudorandom Number Generators for Cryptographic Applications", Information Technology Laboratory, National Institute of Standards and Technology, Gaithersburg, 2010.

[18] P. Z. Wieczorek, "Lightweight TRNG Based on Multiphase Timing of Bistables," IEEE Transactions on Circuits and Systems I: Regular Papers, vol. 63, pp. 1043-1054, 2016.

[19] Y. Liu, R. C. C. Cheung, and H. Wong, "A Bias-Bounded Digital True Random Number Generator Architecture," IEEE Transactions on Circuits and Systems I: Regular Papers, vol. 64, pp. 133-144, 2017.

[20] S. G. Bae, Y. Kim, Y. Park, and C. Kim, "3-Gb/s High-Speed True Random Number Generator Using CommonMode Operating Comparator and Sampling Uncertainty of D Flip-Flop," IEEE Journal of Solid-State Circuits, vol. 52, pp. 605-610, 2017.

[21] I. Cicek, A. E. Pusane, and G. Dundar, "An Integrated Dual Entropy Core True Random Number Generator," IEEE Transactions on Circuits and Systems II: Express Briefs, vol. 64, pp. 329-333, 2017.

[22] M. Park, J. C. Rodgers, D. P. Lathrop, "True random number generation using CMOS Boolean chaotic oscillator", Microelectronics Journal 46, pp. 1364-1370, 2015.

[23] M. Falatic, "Modeling Repo", 2016 [online] Available: https://github.com/MartyMacGyver/memristor-modeling

[24] G. D. U. Guler, "Maximizing randomness in ring oscillators for security applications," in Circuit Theory and Design (ECCTD), 2011 20th European Conference on, 2011, pp. 118-121.

[25] S. Robson, "A Ring Oscillator Based Truly Random Number Generator," University of Waterloo, Waterloo, Ontario, Canada, 2013.

[26] B. Sunar, W. J. Martin, and D. R. Stinson, "A Provably Secure True Random Number Generator with Built-In Tolerance to Active Attacks," IEEE Trans. Comput., vol. 56, pp. 109-119, 2007.

[27] A. A. Abidi, "Phase noise and jitter in cmos ring oscillators," IEEE Journal of Solid-State Circuits, vol. vol. 41, no. 8, pp. pp. 1803-1816, Aug. 2006.

[28] M. Mahvash and A.C. Parker, "A Memristor SPICE Model for Designing Memristor Circuits', in Proc. 53 ${ }^{\text {rd }}$ IEEE International Midwest Symposium on Circuits and Systems (MWSCAS), pp. 989-992, 2010.

[29] R. Marani, G. Gelao and A. G. Perri, “A Review on Memristor Application”, Electronic Devices Laboratory, Polytechnic University of Bari, Bari, Italy, 2015.

[30] M. T. Arafin, C. Dunbar, G. Qu, N. Mcdonald, L. Yan, "A Survey on Memristor Modeling and Security Applications”, 16th Int'l Symposium, ECE Department, University of Maryland College Park, MD, USA, 2015 


\section{BIOGRAPHIES OF AUTHORS}

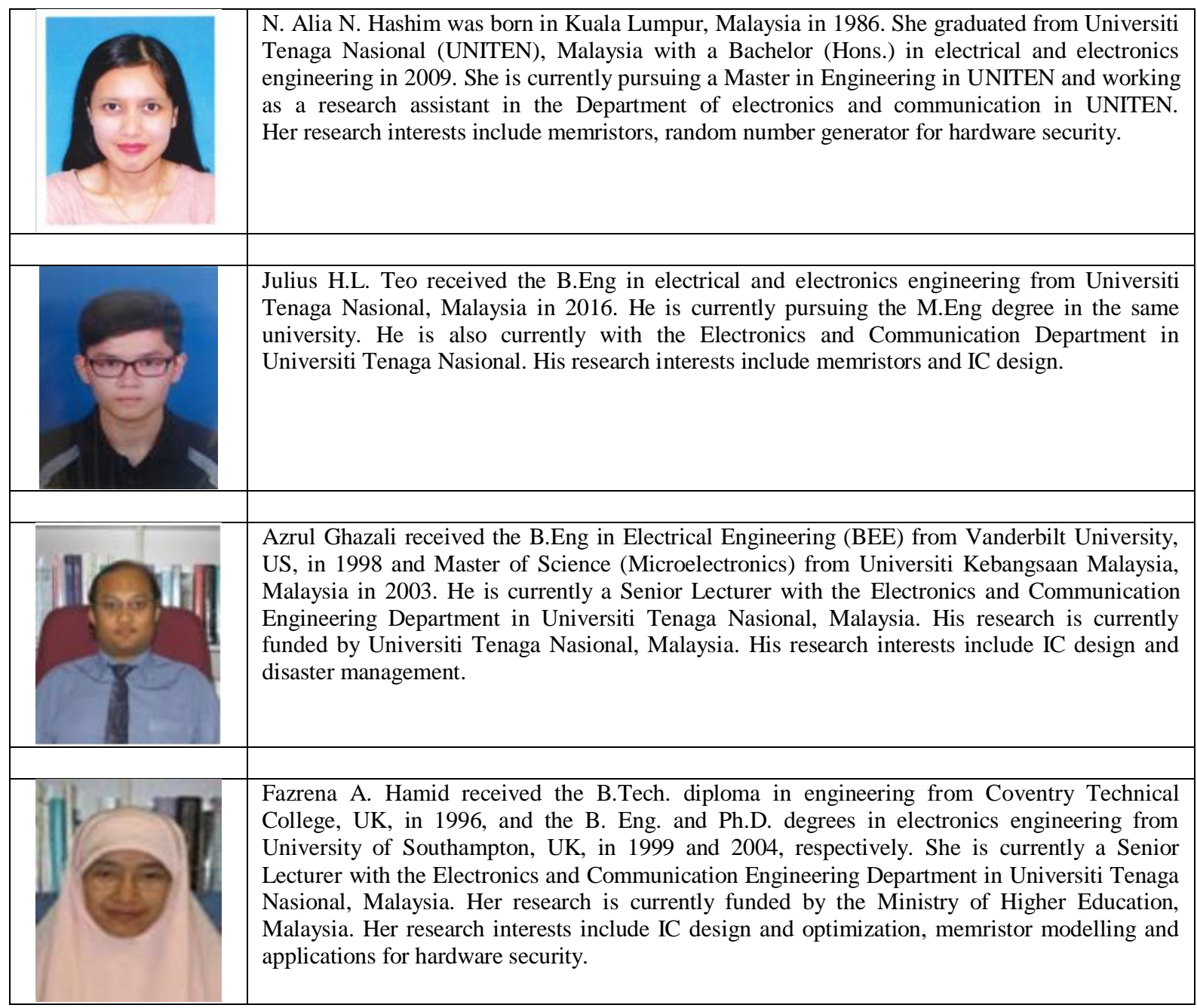

